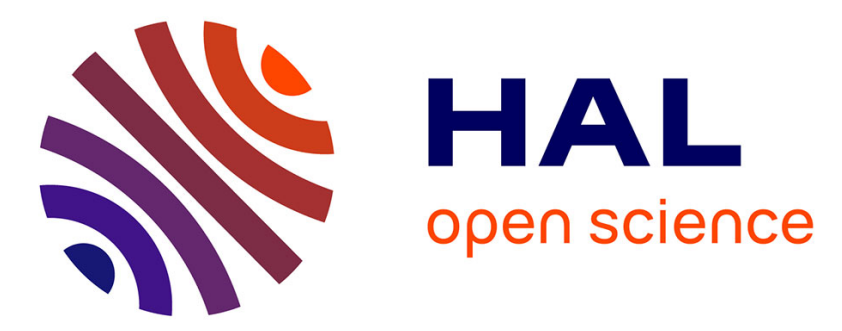

\title{
Defect creation and Diffusion under electric fields from first-principles: the prototypical case of silicon dioxide
}

\author{
N. Salles, L. Martin-Samos, S. de Gironcoli, L. Giacomazzi, M. Valant, Anne \\ Hémeryck, P. Blaise, B. Sklenard, N. Richard
}

\section{To cite this version:}

N. Salles, L. Martin-Samos, S. de Gironcoli, L. Giacomazzi, M. Valant, et al.. Defect creation and Diffusion under electric fields from first-principles: the prototypical case of silicon dioxide. 2019 International Conference on Simulation of Semiconductor Processes and Devices (SISPAD), Sep 2019, Udine, Italy. pp.1-4, 10.1109/SISPAD.2019.8870555 . hal-02338759

HAL Id: hal-02338759

https://hal.laas.fr/hal-02338759

Submitted on 5 Nov 2019

HAL is a multi-disciplinary open access archive for the deposit and dissemination of scientific research documents, whether they are published or not. The documents may come from teaching and research institutions in France or abroad, or from public or private research centers.
L'archive ouverte pluridisciplinaire $\mathbf{H A L}$, est destinée au dépôt et à la diffusion de documents scientifiques de niveau recherche, publiés ou non, émanant des établissements d'enseignement et de recherche français ou étrangers, des laboratoires publics ou privés. 


\section{Defect creation and Diffusion under electric fields from first-principles: the prototypical case of silicon dioxide}

\author{
N. Salles, ${ }^{1,3}$ L. Martin-Samos, ${ }^{1}$ S. de Gironcoli, ${ }^{2}$ L. \\ Giacomazzi, ${ }^{1,3}$ M. Valant, ${ }^{3}$ A. Hemeryck, ${ }^{4}$ P. Blaise, ${ }^{5}$ B. \\ Sklenard, ${ }^{5}$ N. Richard ${ }^{6}$ \\ ${ }^{1}$ CNR-IOM/Democritos National Simulation Center, Istituto \\ Officina dei Materiali, c/o SISSA, via Bonomea 265, \\ IT-34136 Trieste, Italy. ${ }^{2}$ SISSA, via Bonomea 265, IT-34136 \\ Trieste, Italy. ${ }^{3}$ Materials Research Laboratory, University of \\ Nova Gorica, Vipavska 11c 5270-Ajdovščina, Slovenija. \\ ${ }^{4}$ LAAS-CNRS, Université de Toulouse, CNRS, Toulouse, \\ France ${ }^{5}$ Univ. Grenoble Alpes, CEA, LETI, F-38000 \\ Grenoble, France ${ }^{6}$ CEA, DAM, DIF, F-91297 Arpajon, \\ France. \\ email: nsalles33@gmail.com \\ Abstract-In this paper we study the effect on the electric fields \\ on the formation of bulk Frenkal Pairs and on the migration of \\ oxygen interstitials, $I_{O}$, and oxygen vacancies, $V_{O}$, within the \\ framework of Density Functional Theory and Modern Theory of \\ Polarization. At typical OXRRAM field conditions, We show that \\ a significant effect of the electric field is observed only for charged \\ defect. Analyzing the polarization work, we found anomalously \\ high polarization work, for the case of $I_{O}^{-2}$, with respect to the \\ classical picture of the electric work of an isolated point charge. \\ This large difference has to be ascribed to collective contributions \\ coming from the environment.
}

\section{INTRODUCTION}

Resistive switching phenomena taking place under electric fields on oxide-based memories have attracted much attention in the last decade. Under the modelling view point, a number of investigations have exploited empirical kinetic Monte Carlo (kMC) approaches [1], [2], [3], [4], [5] to model ionic transport. In all these approaches, the effect of the electric field on the migration energy change is accounted by means of the classical electric work of a point charge, $Q$ in vacuum, first proposed by Cabrera and Mott [6], as part of their Model of Oxidation of Metals. In other words, the migration enthalpy variation (the polarization work, $W$, at the saddle point of the minimal energy path of a given migration mechanism) is approximated by:

$$
W=\frac{Q d}{2} \cdot \mathcal{E}
$$

The saddle point is assumed to lie half way $(d / 2)$ between the initial and final configuration and the polarization variation $(Q d / 2)$ is implicitly assumed to be co-linear with the applied electric field and the jumping coordinate.
In this work we will present a study on the effect of electric fields in the formation of Frenkel Pairs (VOIO) and in the migration of oxygen vacancies (VO) and interstitials (IO) in $\mathrm{SiO} 2$ from first-principles. We show that, at typical working field conditions in OXRRAM, the polarization work is negligible for neutral defects, i.e. for $\mathrm{V}_{O}^{0}, \mathrm{I}_{O}^{0}$ and the Frenkel pair $\mathrm{V}_{O}^{0} \mathrm{I}_{O}^{0}$. For the same conditions, the contribution to the switching capacity can not be ascribe to the formation of bulk Frenkel pairs (CFP) composed by a $\mathbf{V}_{O}^{+2}$ and a $\mathbf{I}_{O}^{-2}$. An order of magnitude higher field is necessary to trigger a significant contribution from CFP formation. In addition, we find an anomalously high polarization work if compared to the classical electric work of a point charge in vacuum. We demonstrate that this difference has to be ascribed to collective contributions coming from the environment: the movement of the Migrating Atom (MA) is accompanied by the recoil of neighboring atoms such increasing the dipole moment. The proposed new point of view, provides a theoretically grounded framework to the classical "electric work approximation", Equation 1, in which its success, and the success of similar simplified classical single-moving charge pictures can be traced back to subtle system-dependent and symmetryrelated compensations between different effects.

\section{Computational Details}

In our work we use Density Functional Theory (DFT) as in implemented in Quantum Espresso [7], [8]). A PerdewZunger[9] exchange-correlation functional together with a Norm-conserving pseudo-potential from the original QE library ${ }^{1}$ have been used. The valence wave functions are expanded into plane waves basis up to an energy cutoff of 80 Ry. The Brillouin zone is sampled only at $\Gamma \mathrm{k}$-point. For each studied defect, the migration/reaction path is decomposed into a series of intermediate configuration frames. The $\mathrm{SiO}_{2}$, Quartz model is a supercell of 216 atoms with group symmetry $\mathrm{P} 3{ }_{2} 21$. The model has been fully relaxed with a force and stress convergence threshold of $7.10^{-3} \mathrm{eV} / \AA$. Initial and final defect configurations have been relaxed with the same force convergence threshold. This chain-of-frames is, then, optimized (Climbing Image Nudged Elastic Band [10], [11] (NEB) algorithm) to find the minimal energy path. The frame with the highest energy is the saddle point and this energy corresponds to the migration/reaction energy (see Figure 3 for

\footnotetext{
${ }^{1}$ https://www.quantum-espresso.org
} 
a summary of some of the migration/formation mechanisms considered in this work). The Modern Theory of Polarization (MTP) [12], [13] is used to compute the electric enthalpy, macroscopic polarization changes, Born Effective Charges and the polarization work.

\section{RESULTS}

\section{A. Structure and Formation}

$\mathrm{SiO}_{2}$ polymorphs (excluding high-pressure phases) are made of connected $\mathrm{SiO}_{4}$ tetraedra. The building blocks of oxygen Frenkel pairs in silica are the oxygen vacancy $\left(\mathrm{V}_{O}\right)$ and the oxygen interstitial $\left(\mathrm{I}_{O}\right)$. Neutral Frenkel Pairs, NFP, can be formed either by the formation of the neutral defects pairs $\left(\mathrm{V}_{O}^{0}\right.$ and $\left.\mathrm{I}_{O}^{0}\right)$ either by the formation of their respective charged counterparts $\left(\mathrm{V}_{O}^{+2}\right.$ and $\left.\mathrm{I}_{O}^{-2}\right)$, CFP. $\mathrm{I}_{O}^{0} \mathrm{~s}$ have the structure of a peroxide bridge. $\mathrm{V}_{O}^{0} \mathrm{~s}$ are formed by the covalent bonding of two silicon atoms belonging to two different tetraedra ( $\mathrm{Si}-\mathrm{Si}$ bond). Oxygen interstitials with charge -2 have the structure of a twofold Si-O-Si-O ring with pentacoordination for the silicon atoms involved in the ring. For the case of the charge +2 oxygen vacancy, two separated units can be identified, both made from a back-projected positively charged silicon atom weakly bonded to a three-coordinated back oxygen.

Figure 1 and Figure 2 shows the reaction path as a function of the electric field direction, for the formation of firstneighbour Frenkel Pair defects from a chemically ideal -Si-OSi-. Because of the local symmetry, three non-equivalent FP can be formed. That is, there are three different first-neighbour positions for the vacancy and the interstital, with, therefore, different local environments. The dispersion between formation energies is of the order of few tens of electron volts, see Table I, in both the NFP and the CFP. The formation of a NFP requires about $7 \mathrm{eV}$, while the formation of a CFP only 4 $\mathrm{eV}$. The difference between this two quantities is the coulomb interaction energy, that is attractive, between the negative and positive charges of the CFP. As can be seen by comparing Figure 1 with Figure 2, the electric field is only affecting the formation of CFP, with enthalpy variations of few tens of $\mathrm{eV}$. Therefore, at typical working conditions in OXRRAMs, the field intensity is not sufficient to significantly trigger the formation of bulk FP.

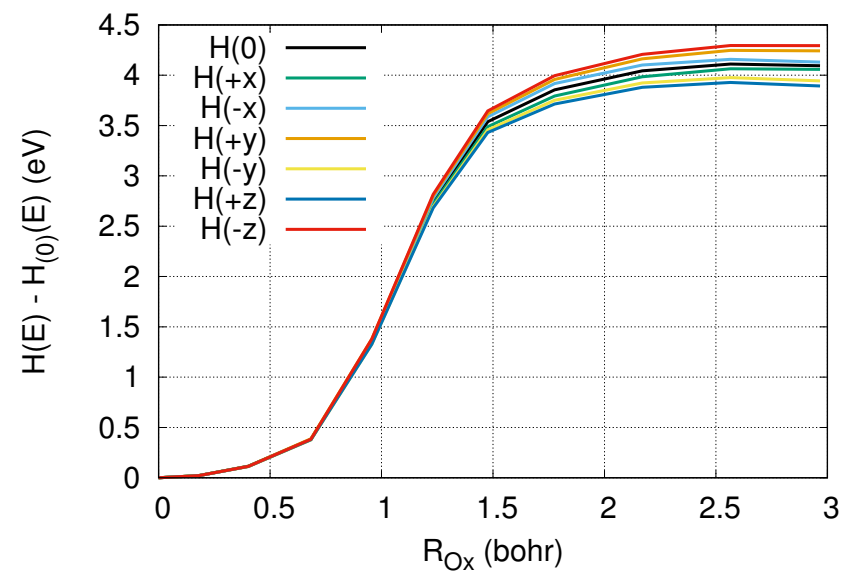

Fig. 1: Formation path of a charged Frenkel pair (CFP), $\mathrm{V}_{O}^{+2} \mathrm{I}_{O}^{-2}$, from a -Si-O-Si-O- quartz structure. Different colors address the enthalpy variation along the path when an electric field of 3.6 $\mathrm{MV} . \mathrm{cm}^{-1}$ is applied along different directions ( \pm orthogonal axis of the box).

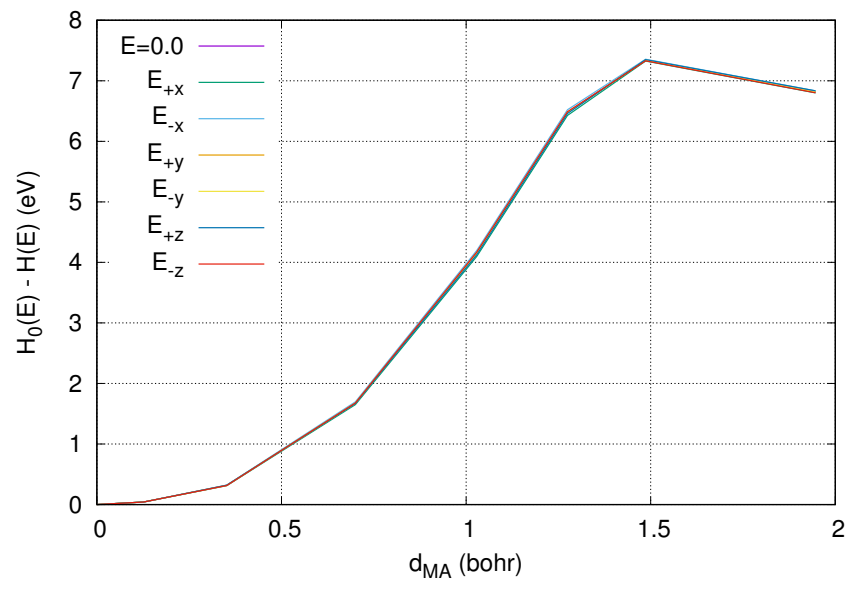

Fig. 2: Formation path of a neutral Frenkel pair (FP), $\mathrm{V}_{O} \mathrm{I}_{O}$, from a -Si-O-Si-O- quartz structure. Different colors address the enthalpy variation along the path when an electric field of $3.6 \mathrm{MV} . \mathrm{cm}^{-1}$ is applied along different directions ( \pm orthogonal axis of the box).

\begin{tabular}{|c|c|c|c|c|c|c|}
\hline & \multicolumn{2}{|c|}{$\mathrm{I}_{O}^{q}$} & \multicolumn{2}{|c|}{$\mathrm{V}_{O}^{q}$} & \multicolumn{2}{|c|}{ FP } \\
\hline $\mathrm{q}(e)$ & 0 & -2 & 0 & +2 & neutral & Charged \\
\hline $\mathrm{E}_{f}(\mathrm{eV})$ & 1.64 & 11.6 & 5.51 & -3.31 & $\begin{array}{c}6.88 \\
7.3 \\
7.1\end{array}$ & $\begin{array}{l}4.86 \\
4.98 \\
4.09\end{array}$ \\
\hline
\end{tabular}

TABLE I: Formation energy of $\mathrm{V}_{O}^{+2}, \mathrm{I}_{O}^{-2}$ and FP for the three nonequivalent first-neighbour positions. The formation energy of isolated defects is computed assuming equilibrium with an $\mathrm{O}_{2}$ reservoir, and an electron reservoir with a Fermi energy the the DFT midgap.

For infinitely far FP defects, the formation energy of the FP is given by the sum of isolated defetcs, column one and two of Table I. The difference between the first-neighbour value and the aforementioned sum of isolate defect is the FP binding energy. The FP binding energy contains both, elastic 
and electrostatic interactions. For the NFP, the sum of isolated defect formation energies gives $7.15 \mathrm{eV}$, very close to the first-neighbour NFP. As the monopolar electrostatic term is zero, the small differences are a measure of the weak elastic interaction between $\mathrm{V}_{O}^{0}$ and $\mathrm{I}_{O}^{0}$. For the CFP the sum of isolated defect formation energy is $8.35 \mathrm{eV}$, significantly larger than the values of $4.86,4.98$ and $4.09 \mathrm{eV}$ computed for firstneighbour FP. The large binding energies, between -4.26 and -3.37 , arise mainly from the large attractive Coulomb between $\mathrm{V}_{O}^{+2}$ and $\mathrm{I}_{O}^{-2}$.

\section{B. Migration of charged vacancies and interstitials}

For the case of the $\mathrm{V}_{O}^{+2}$ migration, the movement of one of the two building units involves the displacement of the backprojected silicon that gets attached to a different neighboring oxygen atom, i.e. the Moving Atom (MA) is a silicon, see Figure 3 a). The twofold ring, that characterize the $\mathrm{I}_{O}^{-2}$, moves by exchanging one of its silicon atom with one of its neighboring silicon through the switching of one of its oxygen atoms, i.e the Migrating Atom (MA) is an oxygen. At the saddle point the aforementioned oxygen presents a dangling bond, see Figure $3 \mathrm{~b}$ ). (a)

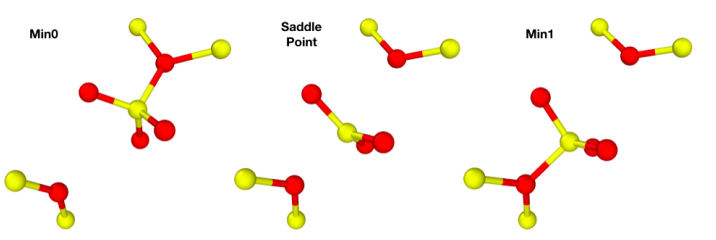

(b)

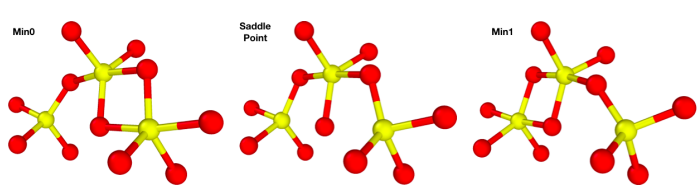

Fig. 3: (a) migration of charged +2 Oxygen Vacancy $\left(\mathrm{V}_{O}^{+2}\right)$, (b) migration of a charged -2 Oxygen Interestitial $\left(\mathrm{I}_{O}^{-2}\right)$.

Along the migration path, the Enthalpy vary proportionally to the polarization work, i.e. between two points, $A$ and $B$, of a reaction path, the work can be written as:

$$
W_{A B}=\int_{A}^{B} \Omega \overrightarrow{d P} \cdot \overrightarrow{\mathcal{E}}=\Omega\left[\overrightarrow{P^{B}}-\overrightarrow{P^{A}}\right] \cdot \overrightarrow{\mathcal{E}}
$$

Figures 5 and 4 show the effect of the polarization work on the migration of $\mathrm{V}_{O}^{+2}$ and $\mathrm{I}_{O}^{-2}$, respectively.

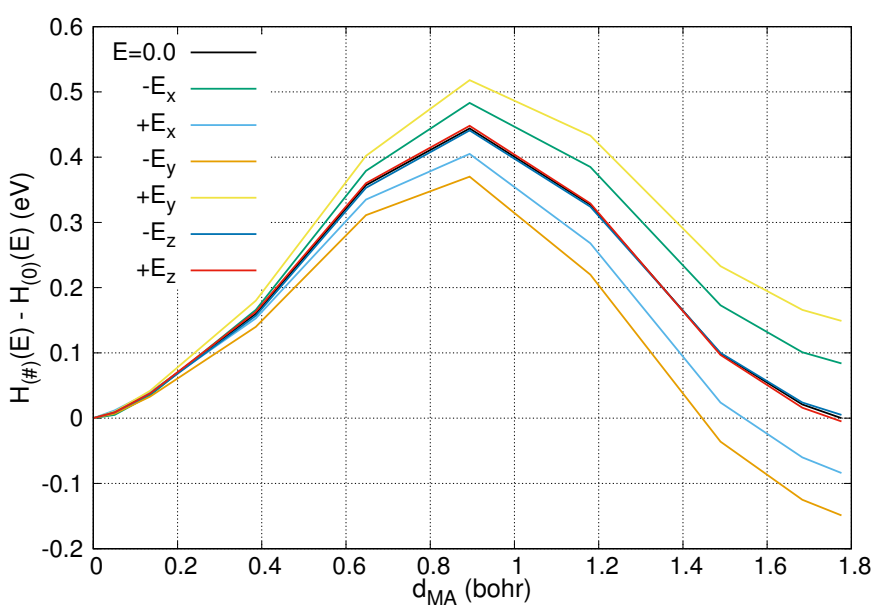

Fig. 4: Energetic path of diffusion of $\mathrm{I}_{O}^{-2}$ under different electric field orientation. Different colors address the enthalpy variation along the path when an electric field of $3.6 \mathrm{MV} . \mathrm{cm}^{-1}$ is applied along different directions ( \pm orthogonal axis of the box).

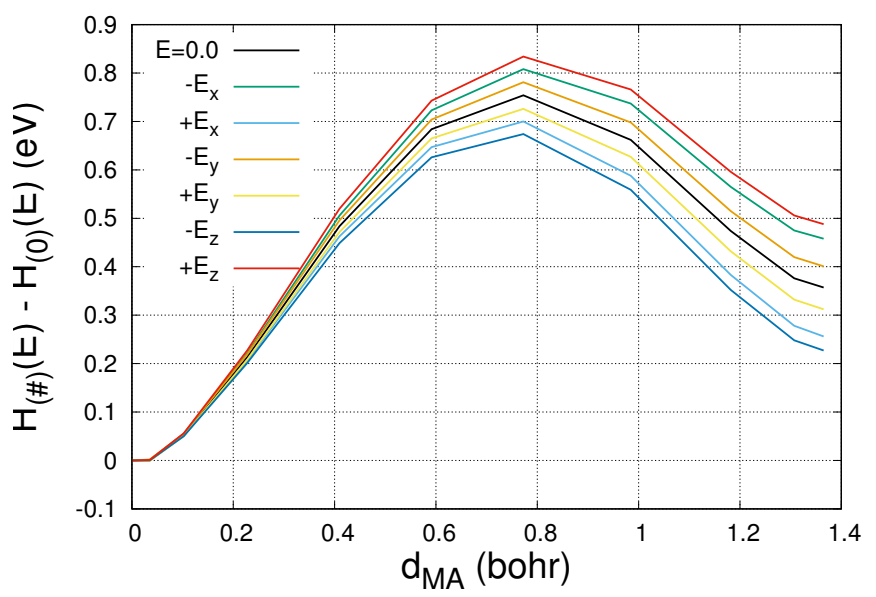

Fig. 5: Energetic path of diffusion of $\mathrm{V}_{O}^{+2}$ under different electric field orientation. Different colors address the enthalpy variation along the path when an electric field of $3.6 \mathrm{MV} . \mathrm{cm}^{-1}$ is applied along different directions ( \pm orthogonal axis of the box).

From initial to Saddle point we find a polarization work for the $\mathrm{V}_{O}^{+2}$ consistent with an equivalent ionic charge of about $+3.4 \mathrm{eV}$, for a jump distance of half $1.36 \mathrm{bohr}$ (see Table II). Such a charge value agrees very well with the Born Effective Charge (BEC) of silicon in $\mathrm{SiO}_{2}$ and with the BEC of a backprojected positively charged silicon atom weakly bonded to a three-coordinated back oxygen. For the $\mathrm{I}_{O}^{-2}$, the Polarization Work Equivalent Charge (PWEC) exceed by more than twice, -4.9 , the $\mathrm{BEC}$ of oxygen in $\mathrm{SiO}_{2}$ or the BEC of oxygens in a charged -2 twofold ring (-2.2), see Table II. With some paperwork, it can be demonstrated that differences between the ionic charge and the first-principles PWEC have to be ascribed to collective contributions coming from the environment, i.e. the MA is accompained by the recoil of environment atoms. Equation 1 can be generalized to explicitly include such collective terms. 


\begin{tabular}{c|c|c|c|c} 
Defect & $\mathrm{d}_{M A}$ & $\left|\Delta \vec{P}_{\|}\right|$ & $\mathrm{Q}$ ionic & $\mathrm{Q}_{\text {eff }}$ \\
\hline $\mathrm{V}_{O}^{+2}$ & 1.46 & 5.19 & 3.4 & 3.4 \\
$\mathrm{I}_{O}^{-2}$ & 0.89 & 4.43 & -2.2 & -4.9 \\
CFP & 1.98 & 17.99 & - & 8.45
\end{tabular}

TABLE II: Distance (in Bohr) traveled by the MA (an oxygen atom for $\mathrm{I}_{O}^{-2}$ and a silicon atom in $\mathrm{V}_{O}^{+2}$ ) up to the saddle point, the macroscopic polarization $\left(\Delta \vec{P}_{\|}\right.$, in units of $e \cdot$ Bohr $)$ at the saddle point, and Born effective charges and effective polarization work charge (in units of $e$ ) for $\mathrm{V}_{O}^{+2}, \mathrm{I}_{O}^{-2}$, and for the CFP formation.

Table III summarizes he key quantities that impact on the defect kinetics. The rate of each event (defect diffusion or defect creation) is computed from Arrhenius law. The magnitude of the effect of polarization work on the rate is relative to the rate without the field. We obtain a factor 4 for the $\mathrm{V}_{O}^{+2}$ and 3 for the $\mathrm{I}_{O}^{-2}$ while a factor hundred for the CFP.

\begin{tabular}{c|c|c|c|c} 
Defect & $\mathrm{E}_{a c}(\mathrm{eV})$ & $\mathrm{W}(\mathrm{eV})$ & $\gamma_{0}$ & $\gamma( \pm \mathcal{E})$ \\
\hline $\mathrm{V}_{O}^{+2}$ & 0.75 & $\begin{array}{l}-0.026 \\
+0.026\end{array}$ & 0.25 & $\begin{array}{c}0.28 \\
0.23\end{array}$ \\
\hline $\mathrm{I}_{O}^{-2}$ & 0.44 & $\begin{array}{l}-0.03 \\
+0.03\end{array}$ & $4 \cdot 10^{4}$ & $\begin{array}{c}1.3 \cdot 10^{5} \\
1.3 \cdot 10^{4}\end{array}$ \\
\hline $\mathrm{CFP}$ & 4.1 & $\begin{array}{l}-0.08 \\
+0.08\end{array}$ & $10^{-57}$ & $\begin{array}{c}10^{-56} \\
10^{-59}\end{array}$
\end{tabular}

TABLE III: Activation energy, polarization work (W) at saddle point for an electric field of $1.8 \mathrm{MV} . \mathrm{cm}^{-1}$, reaction rate (in $s^{-1}$ ) with $\gamma( \pm \mathcal{E})$ and without, $\gamma_{0}$, the electric field for $\left.\mathrm{V}_{O}^{+2}, \mathrm{I}_{O}^{-2}\right)$, and CFP formation. Reaction rates are calculated at room temperature and with an attack frequency of $\nu_{0}=10^{12} \mathrm{~s}^{-1}$.

\section{CONCLUSION}

To summarize, we have performed fisrt-principle calculations, including Modern Theory of Polarization, for the formation of oxygen Frenkel Pairs and for the migration of oxygen vacancies and interstitials. Due to electrostatic interactions the CFP has a formation energy of about $3 \mathrm{eV}$ below the NFP. At typical working field conditions in OXRRAM, the polarization work is negligible for neutral defects, i.e. for $\mathrm{V}_{O}^{0}, \mathrm{I}_{O}^{0}$ and the Frenkel pair $\mathrm{V}_{O}^{0} \mathrm{I}_{O}^{0}$. For the same conditions, the contribution to the switching capacity can not be ascribe to the formation of bulk Frenkel pairs (CFP) composed by a $\mathbf{V}_{O}^{+2}$ and $\mathbf{a} \mathbf{I}_{O}^{-2}$. In addition, we find an anomalously high polarization work resulting in a very high polarization work equivalent charge for $\mathrm{I}_{O}^{-2}$, more than twice the ionic charge of oxygen or its BEC. This difference has to be ascribed to collective contributions coming from the environment: the movement of the Migrating Atom (MA) is accompanied by the recoil of neighboring atoms.

\section{ACKNOWLEDGEMENTS}

High Performance Computing ressources have been provided by the company Arctur (https://hpc.arctur.si) and by the super computing center CINECA (www.cineca.it, grant number ISCRA C HP10CQGOFM). Authors are grateful to stefano Baroni for useful discussions.

\section{REFERENCES}

[1] A. Apolinário, P. Quitério, C. T. Sousa, J. Ventura, J. B. Sousa, L. Andrade, A. M. Mendes, and J. P. Araújo, "Modeling the growth kinetics of anodic $\mathrm{TiO}_{2}$ nanotubes," The Journal of Physical Chemistry Letters, vol. 6, no. 5, pp. 845-851, 2015. PMID: 26262661.

[2] A. J. Choksi, R. Lal, and A. N. Chandorkar, "Growth kinetics of silicon dioxide on silicon in an inductively coupled rf plasma at constant anodization currents," Journal of Applied Physics, vol. 72, no. 4, pp. $1550-1557,1992$.

[3] K. Sasikumar, B. Narayanan, M. Cherukara, A. Kinaci, F. G. Sen, S. K Gray, M. K. Y. Chan, and S. K. R. S. Sankaranarayanan, "Evolutionary optimization of a charge transfer ionic potential model for Ta/Ta-Oxide heterointerfaces," Chemistry of Materials, vol. 29, no. 8, pp. 3603-3614, 2017.

[4] K. S. Martirosyan and M. Zyskin, "Reactive self-heating model of aluminum spherical nanoparticles," Applied Physics Letters, vol. 102, no. 5 , p. $053112,2013$.

[5] F. Raffone and G. Cicero, "Unveiling the fundamental role of temperature in RRAM Switching Mechanism by multiscale simulations," ACS Applied Materials \& Interfaces, vol. 10, no. 8, pp. 7512-7519, 2018. PMID: 29388424

[6] N. Cabrera and N. F. Mott, "Theory of the oxidation of metals," Reports on Progress in Physics, vol. 12, no. 1, p. 163, 1949.

[7] P. Giannozzi, S. Baroni, N. Bonini, M. Calandra, R. Car, C. Cavazzoni, D. Ceresoli, G. L. Chiarotti, M. Cococcioni, I. Dabo, A. D. Corso, S. de Gironcoli, S. Fabris, G. Fratesi, R. Gebauer, U. Gerstmann, C. Gougoussis, A. Kokalj, M. Lazzeri, L. Martin-Samos, N. Marzari, F. Mauri, R. Mazzarello, S. Paolini, A. Pasquarello, L. Paulatto, C. Sbraccia, S. Scandolo, G. Sclauzero, A. P. Seitsonen, A. Smogunov, P. Umari, and R. M. Wentzcovitch, "QUANTUM ESPRESSO: a modular and open-source software project for quantum simulations of materials," Journal of Physics: Condensed Matter, vol. 21, no. 39, p. 395502, 2009.

[8] P. Giannozzi, O. Andreussi, T. Brumme, O. Bunau, M. B. Nardelli, M. Calandra, R. Car, C. Cavazzoni, D. Ceresoli, M. Cococcioni, N. Colonna, I. Carnimeo, A. D. Corso, S. de Gironcoli, P. Delugas, R. A. D. Jr, A. Ferretti, A. Floris, G. Fratesi, G. Fugallo, R. Gebauer, U. Gerstmann, F. Giustino, T. Gorni, J. Jia, M. Kawamura, H.-Y. Ko, A. Kokalj, E. Küçükbenli, M. Lazzeri, M. Marsili, N. Marzari, F. Mauri, N. L. Nguyen, H.-V. Nguyen, A. O. de-la Roza, L. Paulatto, S. Poncé, D. Rocca, R. Sabatini, B. Santra, M. Schlipf, A. P. Seitsonen, A. Smogunov, I. Timrov, T. Thonhauser, P. Umari, N. Vast, X. Wu, and S. Baroni, "Advanced capabilities for materials modelling with Quantum ESPRESSO," Journal of Physics: Condensed Matter, vol. 29, no. 46, p. 465901, 2017.

[9] J. P. Perdew and A. Zunger, "Self-interaction correction to densityfunctional approximations for many-electron systems," Phys. Rev. B, vol. 23, pp. 5048-5079, May 1981.

[10] G. Henkelman and H. Jónsson, "Improved tangent estimate in the nudged elastic band method for finding minimum energy paths and saddle points," The Journal of Chemical Physics, vol. 113, no. 22, pp. 9978-9985, 2000.

[11] G. Henkelman, B. P. Uberuaga, and H. Jónsson, "A climbing image nudged elastic band method for finding saddle points and minimum energy paths," The Journal of Chemical Physics, vol. 113, no. 22, pp. 9901-9904, 2000.

[12] R. Resta, "Macroscopic polarization in crystalline dielectrics: the geometric phase approach," Rev. Mod. Phys., vol. 66, pp. 899-915, Jul 1994.

[13] P. Umari and A. Pasquarello, "Ab initio molecular dynamics in a finite homogeneous electric field," Phys. Rev. Lett., vol. 89, p. 157602, Sep 2002. 